\title{
The impact of COVID-19 lockdown on infants' coronavirus exposure and routine healthcare access in Ireland: the CORAL study at 6 months.
}

Marguerite Lawler ${ }^{1}$, Ruth Franklin ${ }^{1}$, Meredith Kinoshita ${ }^{1}$, Aideen Byrne ${ }^{2}$, John Fitzsimons $^{3}$, Martin White ${ }^{1}$, Naomi McCallion ${ }^{1}$, Liam O Mahony ${ }^{4}$, and Jonathan Hourihane $^{1}$

${ }^{1}$ Royal College of Surgeons in Ireland

${ }^{2}$ Children's Health Ireland at Crumlin

${ }^{3}$ Children's Health Ireland

${ }^{4}$ University College Cork

April 20, 2021

\section{Hosted file}

Lawler m PAIv1.pdf available at https://authorea.com/users/408949/articles/518762-the-impactof-covid-19-lockdown-on-infants-coronavirus-exposure-and-routine-healthcare-access-inireland-the-coral-study-at-6-months 